\title{
5. AOTrauma-Seminar „Niedersachsen Xtra 4“
}

Ulf Culemann

\section{Zum mittlerweile fünften Mal haben Prof. Culemann (Celle), Prof. Gösling (Braun- schweig), Prof. Lehmann (Göttingen) und Prof. Lill (Hannover) das gemeinsame 2-tägige AOTrauma-Seminar „Niedersachsen Xtra4“, diesmal vom 19.01. bis 20.01.2018 in Celle, durchgeführt.}

Die Thematik, die sich mit Frakturen und chronischen Veränderungen der Region „Ellenbogen und Handgelenk" beschäftigte, wurde in 4 größeren Vortrags- und Diskussionsblöcken mit Fallbeispielen unter verschiedenen Gesichtspunkten beleuchtet. Die Themenblöcke wurden jeweils eingeleitet durch die Aufarbeitung der aktuellen Literatur, anschließend dann durch Übersichtsvorträge kompetenter Referenten aufgearbeitet und durch die Diskussion auch schwieriger Fallbeispiele gemeinsam mit den 76 Teilnehmenden bis in die Tiefe hinein bearbeitet. Insbesondere die Behandlung und Nachbehandlung kindlicher Verletzungen des Ellenbogens, aber auch die Frage der operativen Versorgung hochaltriger Patienten spielte in den Vorträgen und Diskussionen immer wieder eine wichtige Rolle und wurde auch von den Teilnehmern sehr hoch in deren Evaluation bewertet. Die Diskussion von Fällen aus der Schlichtungsstelle, die Prof. Oestern aufbereitet hatte, wurde von den Teilnehmenden dann sogar 2 Stunden über die vorgesehene Zeit hinaus intensiv diskutiert. Hier wurden vor allem die besondere Problematik der Patientenführung und das ausführliche Aufklärungsgespräch durch den Behandler deutlich.

Die hohe Teilnehmerzahl aus der Region und die intensiven Diskussionen konnten in Celle einmal mehr zeigen,

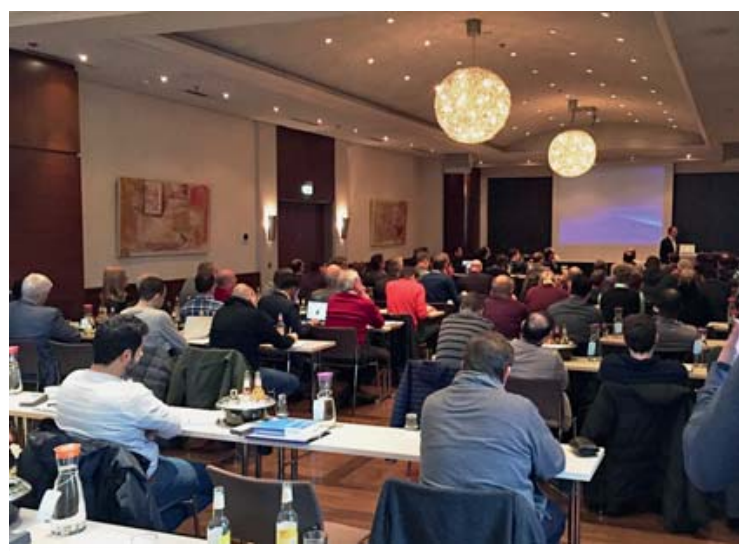

- Abb. 1 Hörsaal. @ Prof. Ulf Culemann dass insbesondere auch der "sektorenüberschreitenden“ Behandlungsführung zwischen den niedergelassenen Kollegen und den im Krankenhaus tätigen Orthopäden und Unfallchirurgen eine wichtige Austauschplattform geboten werden konnte. Die 23 Referenten wurden in Celle wieder sehr stark durch das Publikum gefordert, sie bestätigten aber hinterher, dass durch diesen direkten Austausch auch für die Referenten ein besonderer Stellenwert des Seminars entstand. Die erfreulichen Kommentare der Teilnehmenden und deren gute Bewertungen am Ende des Seminars zeigten aber auch, dass offensichtlich der richtige Mix beim „Xtra4“ getroffen werden konnte. Wir freuen uns auf eine weitere Auflage nächstes Jahr in Hannover, dort wird alle Beteiligten dann die "Schulter intensiv bewegen“.

Prof. Dr. med. Ulf Culemann, Celle

E-Mail: ulf.culemann@akh-celle.de

Bibliografie

DOI https://doi.org/10.1055/a-0591-2710

OP-JOURNAL 2018; 34: 74 ๑ Georg Thieme Verlag KG Stuttgart · New York ISSN 0178-1715

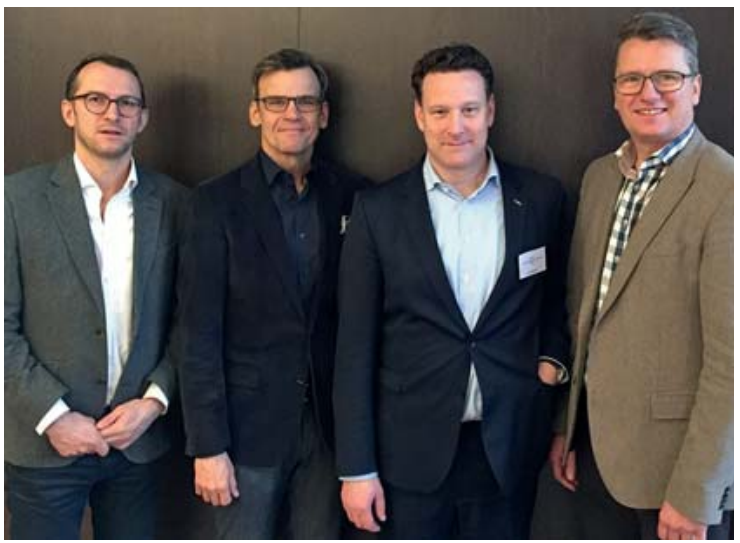

- Abb. 2 Die wissenschaftlichen Leiter Gösling, Lill, Lehmann und Culemann. (c) Prof. Ulf Culemann 